\title{
Determination of Aggregation Numbers of Bile Salt Micelles with the Depression of the Solution Freezing Point
}

\author{
Mihalj Poša* and Kosta Popović
}

\author{
Laboratory of Physical Pharmacy, Department of Pharmacy, Faculty of Medicine, University of Novi Sad, \\ Hajduk Veljkova 3, 21000 Novi Sad, Serbia
}

\begin{abstract}
The special geometry of the steroid skeleton causes formation of bile acid anion micelles with small aggregation numbers, in contrast to aliphatic amphiphiles. Relative to the tendency to reduce membrane toxicity, pharmacological investigations of bile acids are mainly concerned with their oxo derivatives. Since micelles of these bile acids have been insufficiently studied, the objective of this work is the determination of aggregation numbers of corresponding micelle monomers. The aggregation numbers were determined using the freezing point depression of the solutions by applying the appropriate equations of Debye-Hückel, Guggenheim and Gibbs-Duhems, and using pNa data measured with a Na-selective electrode. Depending on the structure of the bile acid anion, the values obtained for the aggregation numbers were in the range from 2.09 to 3.44. The increase in number of oxo groups in the molecule is accompanied by a decrease in hydrophobicity of the convex side of the steroid skeleton of the bile acid anion, resulting in a lower aggregation number.
\end{abstract}

Keywords: Micelles, bile salts, aggregation number, steroid skeleton, critical micellar concentration.

\section{INTRODUCTION}

Bile acid salts are steroid amphiphilic compounds. Bile acid salts or conjugate salts in vertebrates act as biosurfactants. They form mixed micelles with phospholipides (mostly lecithine) which gives them considerable capacity to solubilize cholesterol. Bile salt micelles have an important function in lipid digestion in the small intestine (emulgation and micellar transport) $[1,2]$.

Bile acid salts and their synthesized derivatives have a positive effect on transport of certain medicaments trough cellular lipid barriers, and they are also used as co-surfactants or drug carriers. In the last 20 years biomedical experiments extensively researched sodium salts of oxo derivatives of bile acids, becuse they cause less mambrane toxicity than sodium doxycholate or sodium chenodeoxycholate [37].

Critical micellar concentration (CMC) and aggregation number are important parameters of the micellization process. The less the value of $\mathrm{CMC}$ a certain compound has, the more easily it forms micelles, and it has a negative shift in the Gibbs energy. Relative to the application of micelles as a means to enhance solubility of hydrophobic molecules, it is recommended that the micelle has a large aggregation number value $[1,8]$.

*Address correspondence to this author at the Laboratory of Physical Pharmacy, Department of Pharmacy, Faculty of Medicine, University of Novi Sad, Hajduk Veljkova 3, 21000 Novi Sad, Serbia; Tel: + 38121422 760;

Fax: +381 21422 760; E-mail: mihaljp@uns.ac.rs
Bile acid salt micelles have aggregation numbers ranging from 2 to 16 . It should be considered that overall value of aggregation numbers of most surfactants (alkyl sulfates for example) can be over 100 $[1,8]$.

Aim of this publication is determination of the aggregation numbers of bile acids' oxo derivative sodium salts, since the aggregation numbers for these bile acid salts are not yet known (Figure 1).

\section{MATERIALS AND METHODS}

\subsection{Synthesis of Oxo Derivatives of Cholic, Deoxycholic and Chenodeoxycholic Acids}

Cholic, deoxycholic and chenodeoxycholic acids (Sigma, New Zealand) were used as starting compounds for the synthesis of their oxo derivatives.

$3 \alpha$-Hydroxy-12-oxo-5 $\beta$-cholanoic acid (12-OL) and $3 \alpha, 7 \alpha$-dihydroxy-12-oxo-5 $\beta$-cholanoic acid (12-OCD) were prepared by method used by Miljković et al. [9], while $3 \alpha, 12 \alpha$-dihydroxy-7-oxo-5 $\beta$-cholanoic acid (7ODC) and $3 \alpha$-hydroxy-7-oxo-5 $\beta$-cholanoic acid (7-OL) were obtained by method according to Tullar [10]. 3 $\alpha$ Hydroxy-7, 12-dioxo-5 $\beta$-cholanoic acid (7,12-DOC) was synthesized by a selective oxidation of the $7 \alpha$-hydroxy group of $3 \alpha, 7 \alpha$-dihydroxy-12-oxo- $5 \beta$-cholanoic acid following the procedure of the same author (Tullar). 3 , 12-dioxo-5 $\beta$-cholanoic acid (3,12-DOC) and 3,7-dioxo$5 \beta$-cholanoic acid (3,7-DOC) were obtained by method according to Fieser and Rajagopalan [11] Hyodeoxycholic acid was purchased from Sigma, New Zealand. 


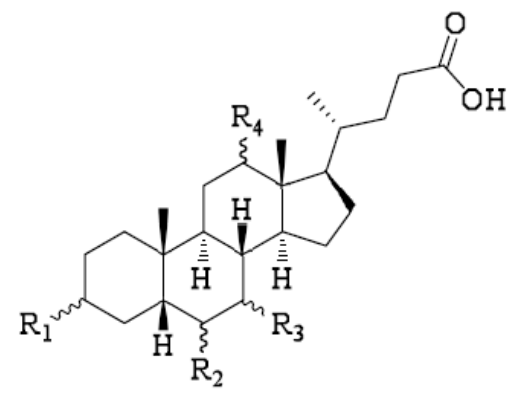

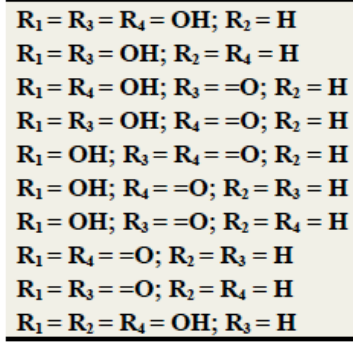

$3 \alpha, 7 \alpha, 12 \alpha$-trihydroxy- $5 \beta$-cholanoic acid (cholic a.); $\mathbf{C}$ $3 \alpha, 7 \alpha$-dihydroxy-5 $\beta$-cholanoic acid (chenodeoxycholic a.); CDC $3 \alpha, 12 \alpha$-dihydroxy-7-oxo-5 $\beta$ cholanoic acid (7-oxodeoxycholic a.); 7-ODC $3 \alpha, 7 \alpha$-dihydoxy-12-oxo-5 $\beta$-cholanoic acid (12-oxochenodeoxycholic a.); 12-OCD $3 \alpha$-hydroxy-7,12-dioxo- $5 \beta$-cholanoic acid; 7,12-DOC $3 \alpha$-hydroxy-12-oxo-5 $\beta$-cholanoic acid (12-oxolithocholic a.); 12-OL $3 \alpha$-hydroxy-7-oxo-5 $\beta$-cholanoic a. (7-oxolithocholic a.); 7-OL 3,12-dioxo-5 $\beta$-cholanoic acid; 3,12-DOC 3,7-dioxo-5 $\beta$-cholanoic acid; 3,7-DOC $3 \alpha, 6 \alpha$-dihydroxy- $5 \beta$-cholanoic acid (hyodeoxycholic acid); HD

Figure 1: Structures of tested bile acids.

\subsection{Determination of Average Aggregation Numbers by Freezing Point Depression}

The determination of the osmolality of bile acids' sodium salts was carried out by measuring the freezing point depression of their solutions relative to water using a cryoscopic osmometer Knauer K-7400. In the measurement, the solution (solvent) was first supercooled without freezing, and then formation of solvent crystals was initiated by vibrations. The osmometer was calibrated using $\mathrm{NaCl}$ solution in the concentration range from 0 to $250 \mathrm{mmol} \mathrm{kg}^{-1}$. Each measurement was repeated five times, and the standard deviation was between $1-2 \%$.

To obtain aggregation numbers of micelles of bile acid anions it is also necessary to have data for concentration of free counter-ion $\left(\mathrm{Na}^{+}, \mathrm{pNa}\right)$ for each measured solution, i.e. to determine the $\mathrm{pNa}$ value for each total concentration of the Na-salt of bile acid. The $\mathrm{pNa}$ values were determined using a Radiometer TitraLab 845 titrator with ion selective electrode ISE21 Na and the reference electrode RedRod201 $(\mathrm{Ag} / \mathrm{AgCl})$ at room temperature. Calibration was carried out with $\mathrm{NaCl}$ solutions.

\section{RESULTS AND DISCUSSIONS}

There are several papers dealing with the issue of polydispersity (particle size) of micellar solutions of bile salts [12-15]. In the determination of aggregation numbers based on osmotic coefficients the polydispersity (relative to particle size) is modeled as a monodisperse micellar system, where the aggregation number corresponds to an average aggregation number $[12,14]$.

In the experiment, changes in the freezing point depression of the solution, the $\Delta T / k$ is followed as a function of the total molal concentration of the bile acid $c_{\mathrm{BA}^{-}}^{t}$. The value $\Delta T / k$ is through the Guggenheim equation (Figure 2. (8)) related to the Bjerrum osmotic coefficient of the solution $\phi$ and to the sum of the molalities of following particles: free monomer (bile acid anion) $c_{\mathrm{BA}^{-}}$, free counterion $c_{\mathrm{C}^{+}}$and of micelle $c_{\mathrm{M}}[16$, 17]. In the region below the break of the function $\Delta T / k=f\left(c_{\mathrm{BA}^{-}}^{t}\right) \quad$ (Figure 3) only free monomers and counter-ions exist (bile acid sodium salts react like strong electrolytes) which means that $\sum_{i} c_{i}$ from the equation (Figure 2. (8)) is $2 c_{\mathrm{BA}^{-}}^{t}$. If we present $\Delta T / k$ $\left(\Delta T / k=2 \phi c_{\mathrm{BA}^{-}}^{t}\right)$ as a function of $\phi c_{\mathrm{BA}^{-}}^{t}$ the line is obtained by the value of the slope 2 , no matter what kind of anion is present [14]. Concentration of bile acids at which the value of $\phi c_{\mathrm{BA}^{-}}^{t}$ has $5 \%$ deviation from the value that corresponds to the line $\Delta T / k=2 \phi c_{\mathrm{BA}^{-}}^{t}$ (i.e. the deviation from the Debye-Hückel law), corresponds to the concentration of the free bile acid anion $c_{\mathrm{BA}^{-}}$ (beginning of the micelle formation). According to Coello et al., in the self-association of bile salts, the $c_{\mathrm{BA}^{-}}$can be assumed to be constant [12, 14]. Table 1 shows the values of $c_{\mathrm{BA}^{-}}$as well as of $c_{\mathrm{BA}^{-}}^{t}$, the 


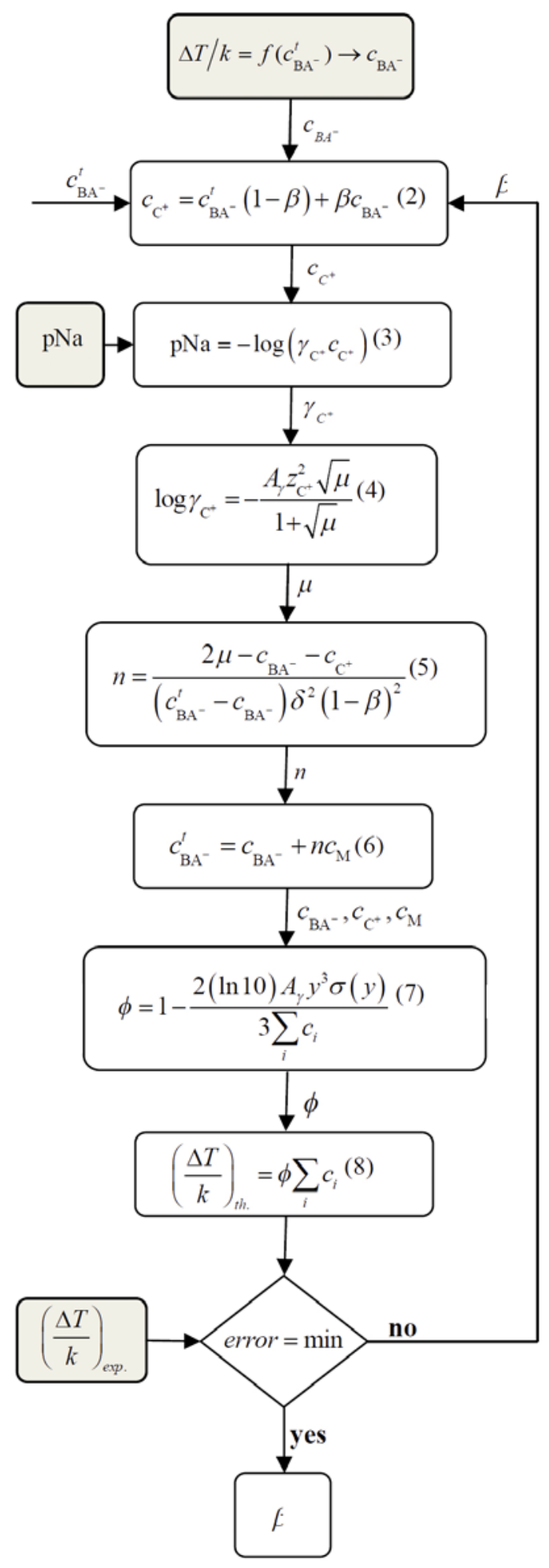

Figure 2: Algorithm for determination of micelle parameters $\left(n\right.$ and $\beta$ ), experimental values are $\mathrm{pNa}$ and $c_{\mathrm{BA}^{-}}$.

concentration corresponding to the break in the function $\Delta T / k=f\left(c_{\mathrm{BA}^{-}}^{t}\right)$ for the investigated sodium salts of bile acid. Many authors assume that the concentration range corresponding to the $\mathrm{CMC}$ of bile salts corresponds to our concentration range: $c_{\mathrm{BA}^{-}}$ $c_{\mathrm{BA}^{-}}^{t}[18,19]$. This is also supported by the literature data for oxo derivatives of bile acid salts [1, 19]. Based on Table 1, it can be concluded that the replacement of $\mathrm{OH}$ groups with oxo groups leads to an increase in the values $c_{\mathrm{BA}^{-}}$and $c_{\mathrm{BA}^{-}}^{t}$, i.e. to a decrease in the hydrophobicity of the steroid nucleus of the bile acid molecule.

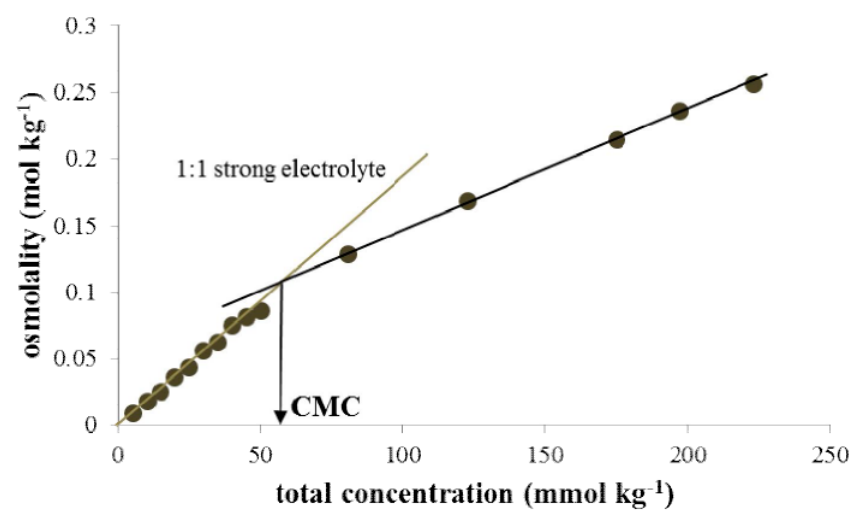

Figure 3: Dependence of $\Delta T / k$ (osmolality) on total concentration $c_{\mathrm{BA}^{-}}^{t}$, example of 7-ODC (the figure shows the upper $\mathrm{CMC}$ value).

Table 1: Ranges of CMC

\begin{tabular}{|c|c|c|}
\hline \multirow{2}{*}{ BA } & \multicolumn{2}{|c|}{$\mathbf{C M C}\left(\mathbf{m m o l}_{\mathbf{~ k g}} \mathbf{- 1}^{-1}\right)$} \\
\cline { 2 - 3 } & $c_{\mathrm{BA}^{-}}$ & $c_{\mathrm{BA}^{-}}^{\mathrm{t}}$ \\
\hline \hline C & 16.4 & 20.5 \\
\hline CDC & 5.0 & 7.5 \\
\hline 7-ODC & 48.5 & 54.8 \\
\hline 12-OCD & 61.4 & 67.5 \\
\hline 7,12-DOC & 105.5 & 125.0 \\
\hline 12-OL & 24.0 & 28.0 \\
\hline 7-OL & 19.7 & 23.0 \\
\hline 3,12-DOC & 79.0 & 93.8 \\
\hline 3,7-DOC & 73.5 & 84.7 \\
\hline HD & 18.3 & 24.5 \\
\hline
\end{tabular}

The aggregation number $n$ and the fraction of bound counterion $\beta$ at a concentration $c_{\mathrm{BA}^{-}}^{t}$ in the micelle region are obtained using the algorithm shown in Figure 2. At some value of $c_{\mathrm{BA}^{-}}^{t}$ which corresponds to the value of $c_{\mathrm{BA}^{-}}$(molal concentration of free bile acid anion) and first approximate value for $\beta$, molal concentration of free counterion $c_{\mathrm{C}^{+}}$is calculated according to equation (Figure 2. (2)). In the next step, 
the experimental pNa value (measured by the ionselective electrode) for the solution $c_{\mathrm{BA}^{-}}^{t}$ is introduced, and then equation (Figure 2. (3)) is used to calculate the activity coefficient of the counterion $\gamma_{\mathrm{C}^{+}}$and, using this quantity, the ionic strength of the solution is obtained from the modified (ion-ion interaction parameter being neglected) Guggenheim equation $\left(A_{Y}=0.4918 \mathrm{~kg}^{1 / 2} \mathrm{~mol}^{-1 / 2}, \quad z_{\mathrm{C}^{+}}=1\right.$ is the charge of the counterion) (Figure 2. (4)). Now, having all variables $(\mu$, $c_{\mathrm{BA}^{-}}, c_{\mathrm{C}^{+}}$and $\beta$ ) known for the given $c_{\mathrm{BA}^{-}}^{t}$ (in the micellar region) it is possible to calculate the aggregation number $(n)$ by equation (Figure 2. (5)) (according to Coello et al., the parameter $\delta$ is 1 [14]). In the next step, with the $n$ also being known, it is possible to determine molal concentration of micelles $c_{\mathrm{M}}$ at the total concentration of bile salt $c_{\mathrm{BA}^{-}}^{t}$ (Figure 2. (6)). Now, with all molal concentrations of all particles in the solution $\left(\sum c\right)$ being known, as well as the ionic strength $\mu$, the Gibbs-Duhem equation is used to calculate the osmotic coefficient of the solution (Figure 2. $(y=\sqrt{\mu}$, $\left.\sigma(y)=3(1+y-1 /(1+y)-2 \ln (1+y)) / y^{3}\right)$. This means that the theoretical value of the freezing point depression $(\Delta T / k)_{\text {th. }}$ (Figure 2. (8)) is calculated, which is then compared with the experimenally obtained value $(\Delta T / k)_{\text {exp. }}$. If the relative error:

$$
\text { error }=\frac{(\Delta T / k)_{\text {exp. }}-(\Delta T / k)_{t h .}}{(\Delta T / k)_{\text {exp. }}}
$$

corresponds to the minimal value, the algorithm stops executing and the micelle parameters $(n$ and $\beta$ ) for $c_{\mathrm{BA}^{-}}^{t}$ are accepted, otherwise the process is continued with the refined $\beta$ values. Table 2 shows obtained values for aggregation numbers $n$ and fractions of bound counterion $\beta$ for investigated micelles. The (average) aggregation numbers for each of the bile acids is constant in the investigated range of concentrations (there are no statistically significant difference between the $n$ values measured at different $c_{\mathrm{BA}^{-}}^{t}$ values). This means that the ratio of the molal fractions in the micelles with different aggregation numbers do not change in the given range of $c_{\mathrm{BA}^{-}}^{t}$. The aggregation number reported for sodium cholate (C) obtained by the same method (freezing point depression) is 3.09 [14], whereas the average value obtained in this work is 3.44. Garidel et al., using also a non-invasive method (isothermal microcalorimetric titration), obtained values from 4.7 to 6.1 in $0.1 \mathrm{M} \mathrm{NaCl}$ for the aggregation number of $\mathrm{Na}$ cholate [20]. A higher value for the aggregation number could be expected because the presence of $\mathrm{NaCl}$ increases the hydrophobicity of bile acids. CMC values as well as the aggregation number depend on the applied method of determination [18]. Aggregation number of bile acid salts obtained by freezing point depression measures between 2 (for oxo derivatives) and 3.5 (for cholic acid). Values obtained by application of $\mathrm{pH}$-metric measurments as well as light diffusion method reach higher numbers, so that the aggregation number value for sodium cholate is above 10. Reason for this can be found in the fact that in other methods for aggregation number determination $n$ values slowly grow with the increase in total monomer concentration [13, 15]. As for the fraction of bound counter-ion $\beta$, it decreases with the decrease in the hydrophobicity of the micelle constituent units (Table 2), i.e. $\beta$ increases with the increase in the aggregation number of the micelle. Coello et al. obtained for $\mathrm{Na}$ cholate an average $\beta$ value of 0.077 [14] whereas we obtained 0.087 . For the $\mathrm{Na}$ salts of oxo derivatives $\beta$ is a negligible quantity (of the total molal concentration of $\mathrm{Na}^{+}$, the average amount bound in the micelle is less than $2 \%$ ), which justifies the Matsuoka-Moroi thermodynamic approach to study of micelles, where the concentration of counterion in the law of mass action is neglected [13, 15].

Researched bile acid sodium salts show that the averige value of $\mathrm{CMC}$ and aggregation number drop with the increase in number of $\mathrm{OH}$ groups supstituted with oxo groups (Figure 4). Oxo group is shifted towards the medium plain of the steroid skeleton (SSMP, Figure 4), so that it nears the concave side of the molecule, which results in the decrease of the hydrophobic surface of steroid skeleton. With the decrease in the hydrophobic surface of the monomer (building block) the contact surphace between the building blocks of the micelles also decreases, which means that the surface of the monomer that is unfavorable for hidratation is getting smaller. At the $\mathrm{CMC}$ value of concentration aggregates are in the form of dimers and correspond to the primary micelles described by Small [1, 18], which gives us the possability to use CMC value as an adequate descriptor for molecular hidrophobicity. From Figure 4. we can observe the linear dependency between $n$ and $\mathrm{CMC}$, which confirms that aggregation number values are greatly influenced by hidrophobicity of the concave side of the steroid skeleton. Cholic acid (C) measurment is an influential observation in the linear reggresion between the aggregation number and $\mathrm{CMC}$, which is a result of the probable hydrogen bonding in the micell $\mathrm{C}$, which makes $\mathrm{C}$ more hydrophobic than expected based on its hydrophobic surface. If cholic 
Table 2: Freezing Point Depression, pNa Values, Deduced Values of Aggregation Numbers and Fractions of Bound Counterions Measured at Different Total Bile Salt Concentration

\begin{tabular}{|c|c|c|c|c|}
\hline$c_{\mathrm{BA}^{-}}^{t}$ & $(\Delta T / k)_{e x .}$ & \multirow{2}{*}{$\mathrm{pNa}$} & \multirow{2}{*}{$n$} & \multirow{2}{*}{$\beta$} \\
\hline \multicolumn{2}{|c|}{$\left(\mathrm{mol} \mathrm{kg}^{-1}\right)$} & & & \\
\hline \multicolumn{5}{|c|}{ C } \\
\hline 0.0455 & 0.0587 & 1.45 & 3.41 & 0.023 \\
\hline 0.0725 & 0.0802 & 1.28 & 3.43 & 0.058 \\
\hline 0.1685 & 0.152 & 0.99 & 3.44 & 0.12 \\
\hline 0.200 & 0.171 & 0.95 & 3.48 & 0.16 \\
\hline \multicolumn{5}{|c|}{ CDC } \\
\hline 0.0483 & 0.0480 & 1.53 & 3.12 & 0.28 \\
\hline 0.1985 & 0.157 & 1.04 & 3.19 & 0.36 \\
\hline \multicolumn{5}{|c|}{ 7-ODC } \\
\hline 0.0807 & 0.124 & 1.21 & 2.65 & 0.018 \\
\hline 0.1225 & 0.162 & 1.07 & 2.75 & 0.025 \\
\hline 0.1750 & 0.214 & 0.93 & 2.59 & 0.017 \\
\hline 0.1972 & 0.232 & 0.88 & 2.64 & 0.023 \\
\hline 0.2230 & 0.247 & 0.84 & 2.82 & 0.028 \\
\hline \multicolumn{5}{|c|}{ 12-OCD } \\
\hline \multicolumn{5}{|c|}{ 7,12-DOC } \\
\hline 0.1407 & 0.234 & 0.99 & 2.05 & 0.017 \\
\hline 0.1620 & 0.271 & 0.94 & 2.08 & 0.018 \\
\hline 0.1892 & 0.288 & 0.88 & 2.12 & 0.019 \\
\hline 0.220 & 0.320 & 0.83 & 2.10 & 0.020 \\
\hline 0.2481 & 0.350 & 0.78 & 2.12 & 0.019 \\
\hline \multicolumn{5}{|c|}{$12-\mathrm{OL}$} \\
\hline 0.0485 & 0.0720 & 1.42 & 2.80 & 0.019 \\
\hline 0.0753 & 0.0979 & 1.25 & 2.78 & 0.022 \\
\hline 0.1255 & 0.155 & 1.06 & 2.83 & 0.018 \\
\hline 0.1706 & 0.181 & 0.95 & 2.83 & 0.021 \\
\hline 0.2150 & 0.219 & 0.86 & 2.82 & 0.023 \\
\hline
\end{tabular}


(Table 2). Continued.

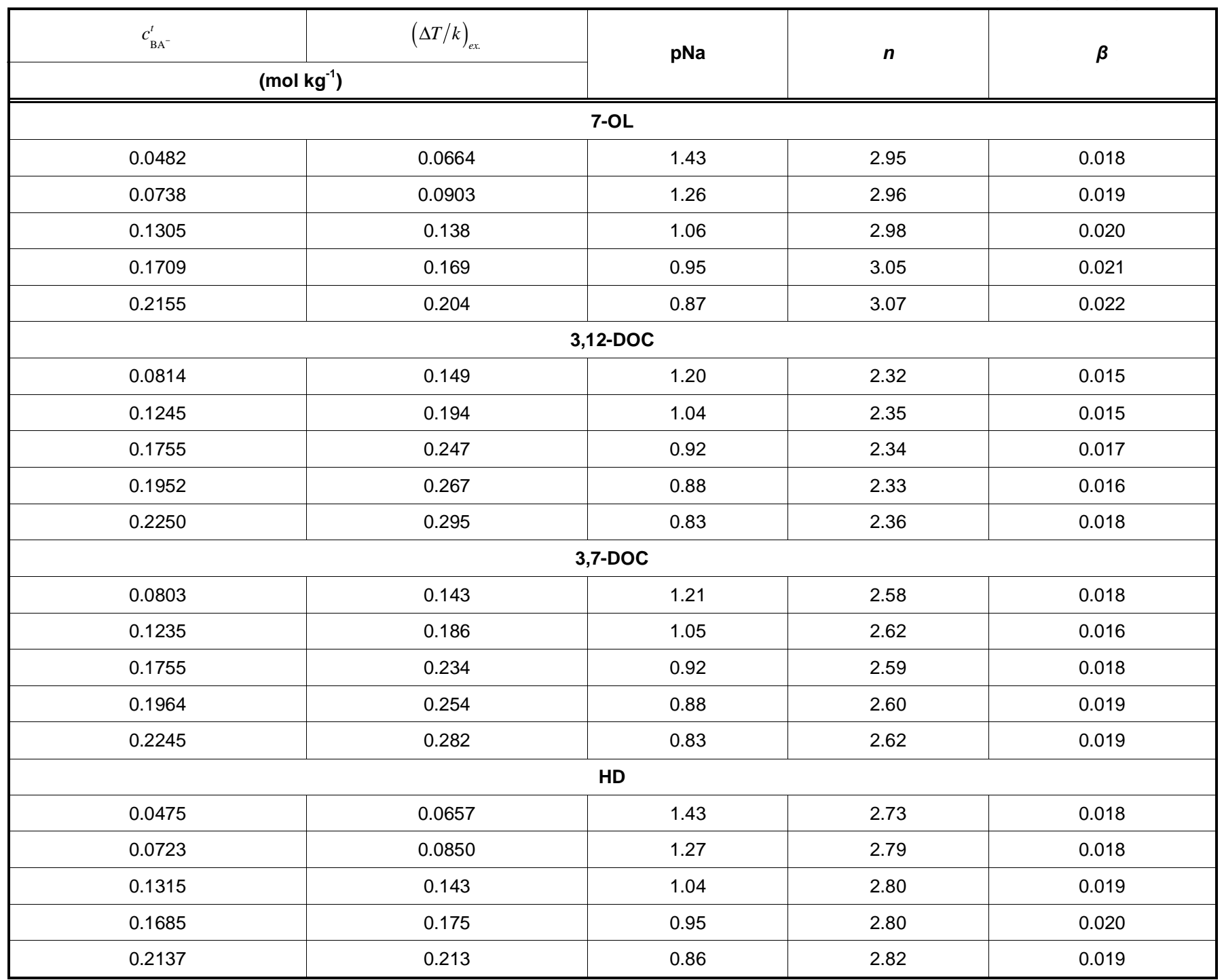

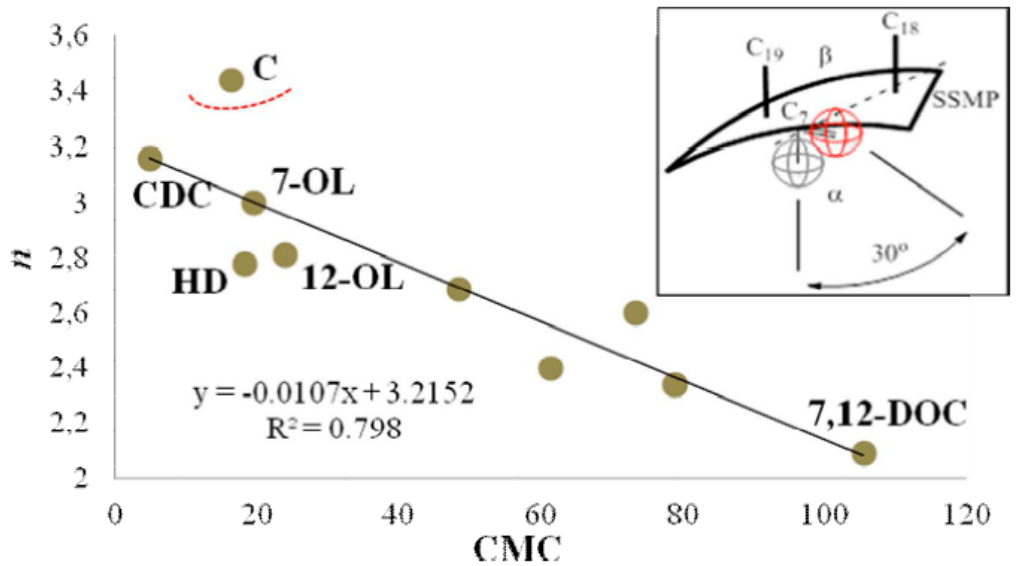

Figure 4: Dependence between average aggregation number and critical micellar concentration.

acid is excluded from the liner regresion, correlation coefficient $n=f(\mathrm{CMC})$ reaches a new value 0.9203 . It is researched that hydrogen bonding is also possible in 7-oxo-deoxycholic acid [19], but it is not visible in Figure 4, which means that 7-oxo-deoxycholic acid sodium salt is not an infuential observation. All this 
shows that freezing point depression determination method is less sensitive or insensitive as far as aggregates such as Small's secondary micelles are concerned.

\section{CONCLUSIONS}

Aggregation number measured with the use of freezing point depression method has values from $\approx 2$ up to $\approx 3.5$, depending on the structure of the steroid skeleton. Between the average value of the aggregation number and the CMC value there exists a linear dependency, which makes us conclude that the aggregation number is also influenced by the available hydrophobic surface on the concave side of the bile acid molecule.

\section{ACKNOWLEDGEMENTS}

This study was financially supported by the Provincial Secretariat for Science and Technological Development, AP Vojvodina, Republic of Serbia, Grant No. 114-451- 2113/2011-02. M.P. also acknowledges to the Domus Hungarian Scholarship (Hungarian Academy of Sciences).

\section{REFERENCE}

[1] Poša M. Hydrophobicity and self-association of bile acids with a special emphasis on oxo derivatives of 5 - $\beta$-cholanic acid. Curr Org Chem 2012; 16: 1876-04. http://dx.doi.org/10.2174/138527212802651304

[2] Calabresi M, Andreozzi P, La Mesa C. Supra-molecular association and polymorphic behaviour in systems containing bile acid salts. Molecules 2007; 12: 1731-54. http://dx.doi.org/10.3390/12081731

[3] Hofmann AF, Roda A. Physicochemical properities of bile acids and their relationship to biological properties: an overview of the problem. J Lipid Res 1984; 25: 1477-89.

[4] Mikov M, Fawcett JP. Bile Acids. 1rd ed., Geneva: Medishet Publisher 2007.

[5] Garidel P, Hildebrand A, Knauf K, Blume A. Membranolytic activity of bile salts: influence of biological membrane properities and composition. Molecules 2007; 12: 2292-26. http://dx.doi.org/10.3390/12102292

[6] Poša M, Kuhajda K. Hydrophobicity and haemolytic potential of oxo derivatives of cholic, deoxycholic and chenodeoxycholic acids. Steroids 2010; 75: 424-31. http://dx.doi.org/10.1016/j.steroids.2010.02.008
[7] Poša M, Farkaš Z. Cholesterol solubilization by oxo derivatives of selected bile acids and their osmotic resistance. Collect Czech Chem Commun 2010; 75: 767-84. http://dx.doi.org/10.1135/cccc2009559

[8] Poša M, Ćirin D. Mixed micelles of sodium salts of bile acids and Tween 40: Effect of the steroid on the coefficient of interaction in mixed micelles. Ind Eng Chem Res 2012; 51 : 14722-28.

http://dx.doi.org/10.1021/ie301648h

[9] Miljković D, Kuhajda K, Hranisavljević J. Selective C-12 oxidation of cholic acid. J Chem Res (S) 1996; 106-107.

[10] Tullar BF, inventor; Hemi-diethylene glycol adduct of 3,12dihydroxy-7-ketocholanic acid and preparation thereof. US Patent 2549 947. 1951.

[11] Fieser LF, Rajagopalan S. Oxidation of Steroids III. Selective oxidations and acylations in bile acid series. J Am Chem Soc 1950; 72: 5530-36. http://dx.doi.org/10.1021/ja01168a046

[12] Coello A, Meijide F, Rodríguez Núñez E, Vázquez Tato J. Aggregation behavior of bile salts in aqueous solution. $J$ Pharm Sci 1996; 85(1): 9-15. http://dx.doi.org/10.1021/js950326

[13] Matsuoka K, Moroi Y. Micelle formation of sodium deoxycholate and. sodium ursodeoxycholate. Biochim Biophys Acta 2002; 1580: 189-99. http://dx.doi.org/10.1016/S1388-1981(01)00203-7

[14] Coello A, Meijide F, Rodríguez Núñez E, Vázquez Tato J. Aggregation behavior of sodium cholate in aqueous solution. J Phys Chem 1993; 97: 10186-91. http://dx.doi.org/10.1021/j100141a046

[15] Ninomiya R, Matsuoka K, Moroi $Y$. Micelle formation of sodium chenodeoxycholate and solubilization into the micelles: comparison with other unconjugated bile salts. Biochim Biophys Acta 2003; 1634: 116-25.

http://dx.doi.org/10.1016/j.bbalip.2003.09.003

[16] Guggenheim EA, Turgeon JC. Specific interaction of ions. Trans Faraday Soc 1955; 51: 747-61. http://dx.doi.org/10.1039/tf9555100747

[17] Coello A, Meijide F, Rodríguez Núñez E, Vázquez Tato JJ. Aggregation behavior of sodium cholate in aqueous solution. J Pharm Sci 1994; 83(6): 828-32. http://dx.doi.org/10.1002/jps.2600830614

[18] Madenci D, Egelhaaf US. Self-assembly in aqueous bile salt solutions. Curr Opin Colloid Interface Sci 2010; 15: 109-15. http://dx.doi.org/10.1016/j.cocis.2009.11.010

[19] Poša M, Guzsvány V, Csanádi J. Determination of critica micellar concentration of two monoketo derivatives of cholic acid. Colloids Surf Biointerfaces 2009; 74: 84-90. http://dx.doi.org/10.1016/j.colsurfb.2009.06.029

[20] Garidel P, Hildebrand A, Neubert, R, Blume A Thermodynamic characterization of bile salt aggregation as a function of temperature and ionic strength using isotermal titration calorimetry. Langmuir 2000; 16: 5267-75. http://dx.doi.org/10.1021/la9912390 